\title{
Superacid-promoted synthesis of polychlorinated dibenzofurans
}

\author{
Ahmad Qarah, ${ }^{a}$ Makafui Gasonoo, ${ }^{\mathrm{a}}$ Dat Do, ${ }^{\mathrm{b}}$ and Douglas A. Klumpp*,a \\ ${ }^{a}$ Department of Chemistry and Biochemistry, Northern Illinois University, DeKalb, IL \\ 60115. 'Department of Chemistry, California State Polytechnic University, Pomona, CA, \\ 91768. \\ dklumpp@niu.edu
}

INDEX

Page $1 \quad$ Index

Page 2-4 Experimental procedures and characterization data.

Pages 4-13 $\quad{ }^{1} \mathrm{H}$ and ${ }^{13} \mathrm{C}$ NMR spectra for compounds 6-11

Page $14 \quad$ Computational details

Pages 14-17 Computed structures 


\section{Experimental section}

All reagents were purchased from commercial suppliers and used as received. However, triflic acid was distilled immediately prior to its use. Products were purified using silica gel chromatography and standard procedures with $100 \%$ hexanes as the mobile phase. ${ }^{17}$ Calculations were done using the Gaussian 09 program suite. ${ }^{18}$ Geometry optimizations were done without constraints at the indicated level of theory. Frequency calculations were done on all structures and each was determined to have zero imaginary frequencies. Energy values were calculated from optimized structures and corrected for zero point energies. Caution! Polychlorodibenzofurans are exceedingly toxic substances. Their handling should only be done by trained individuals with adequate personal protective and safety equipment.

Procedure for the Synthesis of Dibenzofurans. Under an inert atmosphere, a solution is prepared containing triflic acid (1 mL, $11 \mathrm{mmol})$, arene $(1 \mathrm{~g}$, ca. $8 \mathrm{mmol})$, and co-solvent (1 $\mathrm{mL}$ of $\mathrm{CH}_{2} \mathrm{Cl}_{2}$ or $\mathrm{CF}_{3} \mathrm{CH}_{2} \mathrm{OH}$ ) o-Chloranil (0.15 g, $0.6 \mathrm{mmol}$ ). The mixture is cooled to $-30{ }^{\circ} \mathrm{C}$ and a solution of $o$-chloranil 5 (in $2 \mathrm{~mL}$ co-solvent) is added slowly with stirring. The resulting mixture is allowed to slowly warm to room temperature overnight. Following this reaction period, the mixture is poured over ice and the solution is extracted three times with chloroform. The organic extracts are then washed twice with water and twice with brine solution. Following a drying regiment with anhydrous $\mathrm{Na}_{2} \mathrm{SO}_{4}$, the organic solvent is removed and the crude product is subject to chromatography with hexanes.

1,2,3,4-Tetrachlorodibenzo[b,d]furan (6). White solid, mp 164-166 ${ }^{\circ} \mathrm{C}$ (hexanes; lit., 169-170 $\left.{ }^{\circ} \mathrm{C}\right) .{ }^{19}{ }^{1} \mathrm{H}$ NMR ( $\left.\mathrm{CDCl}_{3}, 500 \mathrm{MHz}\right): \delta, 7.43-7.49$ (m, 1H), 7.58-7.63 (m, 1H), 7.65 (d, $J=8.0 \mathrm{~Hz}, 1 \mathrm{H}), 8.33 \mathrm{~d}, J=4.5 \mathrm{~Hz}, 1 \mathrm{H}) .{ }^{13} \mathrm{C} \mathrm{NMR}\left(\mathrm{CDCl}_{3}, 125 \mathrm{MHz}\right): \delta, 112.7$, 116.5, 121.4, 122.0, 123.6, 124.9, 125.6, 127.8, 131.0, 135.0, 150.9, 156.6. Low resolution MS (EI), 308/306/304 (M+), 243/241, 206, 171/173, 153, 121. High resolution mass spectrum (EI), calcd for $\mathrm{C}_{12} \mathrm{H}_{4} \mathrm{Cl}_{4} \mathrm{O}, 303.9016$, found, 303.9013. 
1,2,3,4,7-Pentachlorodibenzo[b,d]furan (7). White solid, mp 153-155 ${ }^{\circ} \mathrm{C}$ (hexanes). ${ }^{1} \mathrm{H}$ NMR ( $\mathrm{CDCl}_{3}, 300 \mathrm{MHz}$ ): $\delta, 7.35$ (dd, $J=1.8,8.4 \mathrm{~Hz}, 1 \mathrm{H}$ ), 7.58 (d, $J=1.8 \mathrm{~Hz}, 1 \mathrm{H}$ ), 8.16 (d, $J=8.4 \mathrm{~Hz}, 1 \mathrm{H}) .{ }^{13} \mathrm{C}$ NMR $\left(\mathrm{CDCl}_{3}, 75 \mathrm{MHz}\right): \delta, 113.0,116.2,123.7,124.7,125.9$, 127.7, 131.1, 135.1, 150.8, 156.5. Low resolution MS (EI), 344/342/340/338 (M+), 279/277/275, 205/207, 170, 138, 120. High resolution mass spectrum (EI), calcd for $\mathrm{C}_{12} \mathrm{H}_{3} \mathrm{Cl}_{5} \mathrm{O}$, 337.8627, found, 337.8635 .

1,2,3,4,7,8-Hexachlorodibenzo[b,d]furan (8; obtained as a mixture with 9). ${ }^{1} \mathrm{H}$ NMR

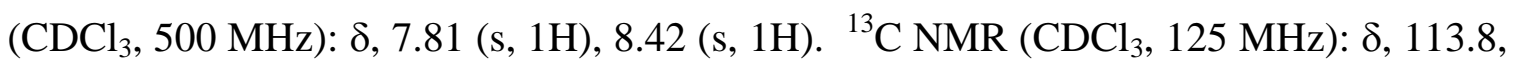
121.3, 122.6, 123.8, 128.5, 132.0, 133.5, 151.2, 154.7. Low resolution MS (EI), 378/376/374/372 (M+), 313/311/309, 243/241/239, 187, 155, 138. High resolution mass spectrum (EI), calcd for $\mathrm{C}_{12} \mathrm{H}_{2} \mathrm{Cl}_{6} \mathrm{O}, 371.8237$, found, 371.8236 .

7-Bromo-1,2,3,4-Tetrachlorodibenzo[b,d]furan (10). White solid, mp 202-205 ${ }^{\circ} \mathrm{C}$ (hexanes). ${ }^{1} \mathrm{H} \mathrm{NMR}\left(\mathrm{CDCl}_{3}, 300 \mathrm{MHz}\right): \delta, 7.52$ (dd, $\left.J=5.7,1.2 \mathrm{~Hz}, 1 \mathrm{H}\right), 7.77$ (d, $J=1.2$ $\mathrm{Hz}, 1 \mathrm{H}), 8.11$ (d, $J=5.7 \mathrm{~Hz}, 1 \mathrm{H}) .{ }^{13} \mathrm{C} \mathrm{NMR}\left(\mathrm{CDCl}_{3}, 125 \mathrm{MHz}\right): \delta$, 115.6, 116.6, 121.8, 122.1, 122.6, 123.9, 125.6, 127.6, 127.8, 127.8, 131.2, 150.8, 156.6. Low resolution MS (EI), 388/386/384/382 (M+), 279/277/275, 207/205, 192, 160, 135, 120, 102, 85. High resolution mass spectrum (EI), calcd for $\mathrm{C}_{12} \mathrm{H}_{3} \mathrm{BrCl}_{4} \mathrm{O}$, 381.8121, found, 381.8111 .

7-Fluoro-1,2,3,4-Tetrachlorodibenzo[b,d]furan (11). White solid, mp $176-178{ }^{\circ} \mathrm{C}$ (hexanes). ${ }^{1} \mathrm{H}$ NMR (CDCl, $300 \mathrm{MHz}$ ): $\delta, 7.22$ (dt, $J=2.5,9.0 \mathrm{~Hz}, 1 \mathrm{H}$ ), 7.38 (dd, $J=2.5$, $8.5 \mathrm{~Hz}, 1 \mathrm{H}$ ), 8.29 (dd, $J=5.5,9.0 \mathrm{~Hz}, 1 \mathrm{H}) .{ }^{13} \mathrm{C} \mathrm{NMR}\left(\mathrm{CDCl}_{3}, 125 \mathrm{MHz}\right): \delta, 100.1$ (d, $J_{C-}$ $\left.F_{F}=26.3 \mathrm{~Hz}\right), 112.4\left(\mathrm{~d}, J_{C-F}=23.8 \mathrm{~Hz}\right), 116.4,119.0,122.2,124.0\left(\mathrm{~d}, J_{C-F}=2.5 \mathrm{~Hz}\right)$, 125.4, 127.7, 130.4, 151.2, 156.9 (d, $\left.J_{C-F}=12.5 \mathrm{~Hz}\right), 163.2\left(\mathrm{~d}, J_{C-F}=248 \mathrm{~Hz}\right)$, Low resolution MS (EI), 326/324/322 (M+), 263/261/259, 191/189, 162, 130, 112. High resolution mass spectrum (EI), calcd for $\mathrm{C}_{12} \mathrm{H}_{3} \mathrm{FCl}_{4} \mathrm{O}$, 321.8922, found, 321.8925 . 
Procedure for the Chlorination of Dibenzofurans 6 and 8/9. The dibenzofuran (ca. $0.050 \mathrm{~g}, 0.2 \mathrm{mmol}$ ) is dissolved $1 \mathrm{~mL}$ of $\mathrm{CHCl}_{3}$ and $\mathrm{N}$-chlorosuccinimide $(0.16 \mathrm{~g}, 1.2$ mmol) is added. To this solution, $2 \mathrm{~mL}(23 \mathrm{mmol})$ of triflic acid is added. The mixture is stirred for $24 \mathrm{hr}$ at room temperature, after which it is poured over about $10 \mathrm{~g}$ of ice. The aqueous phase is extracted twice with $\mathrm{CHCl}_{3}$ and the combined organic extracts are then washed with water and a brine solution (twice). The mixture is dried with sodium sulfate and analyzed by GC-MS and GC-FID.

Results from chlorination of compound 6 (relative yields):
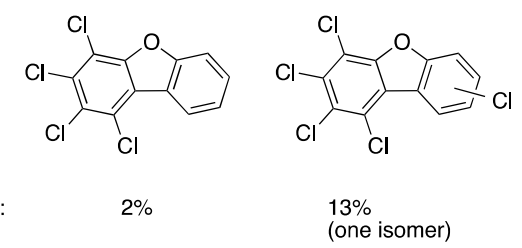

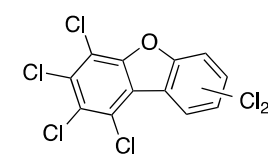

$38 \%$ and $9 \%$
(two isomers)

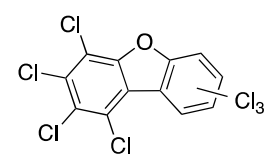

$24 \%$ and $8 \%$ (two isomers)

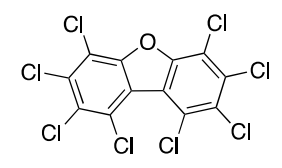

$7 \%$

Results from chlorination of compound $\mathbf{8}$ (relative yields):

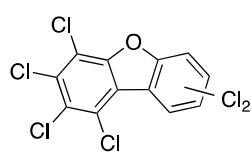

Product Distribution:

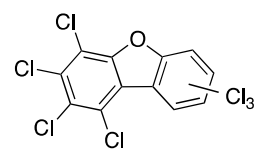

$14 \%$ and $8 \%$

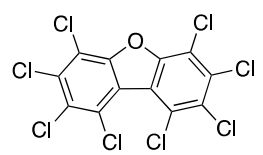

$4 \%$ 


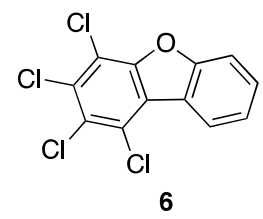

${ }^{1} \mathrm{H}$ NMR

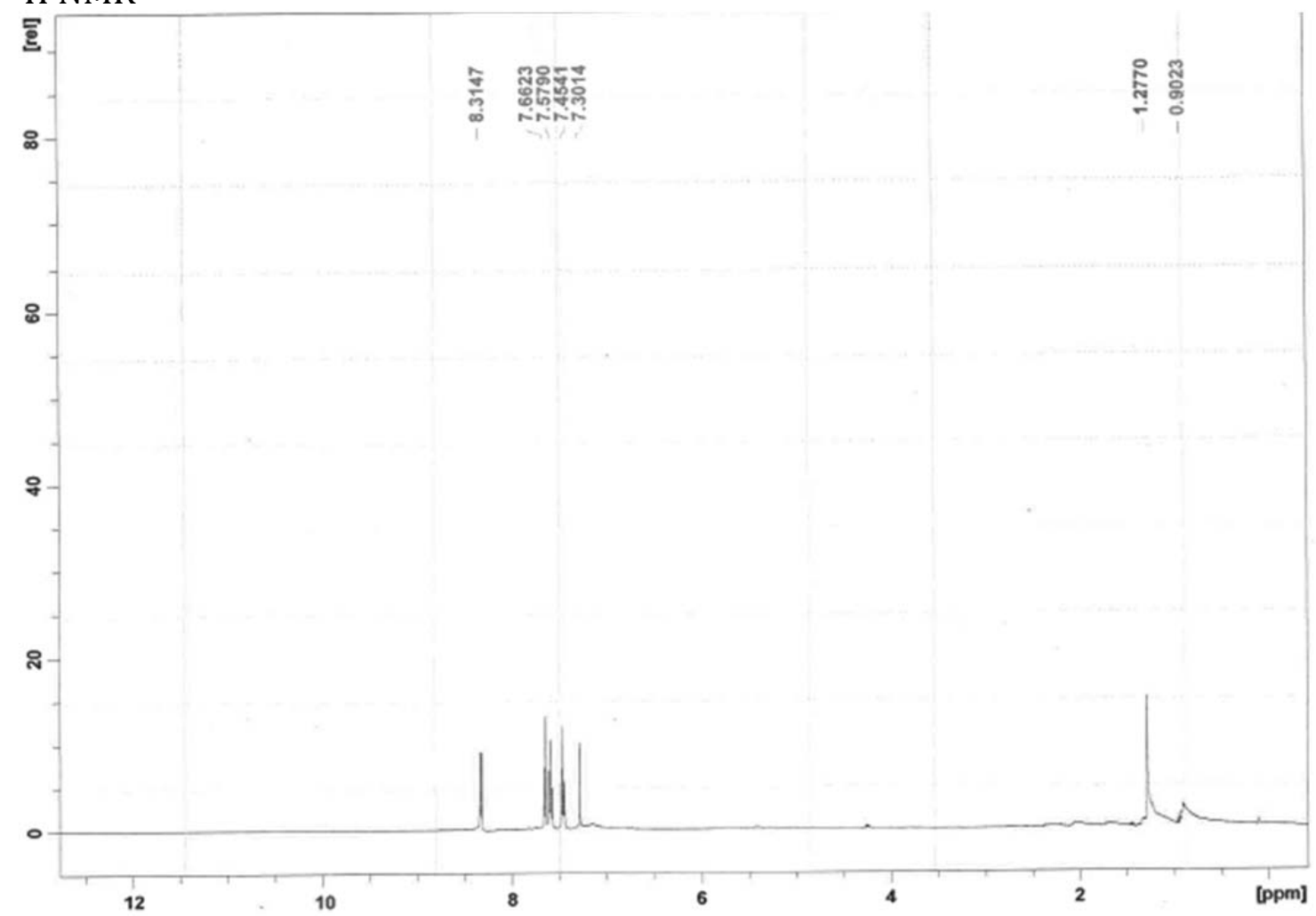




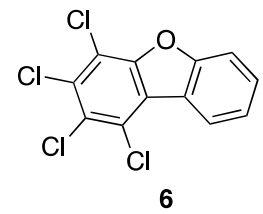

${ }^{13} \mathrm{C} \mathrm{NMR}\left(\mathrm{CDCl}_{3}\right.$ with acetone impurity)

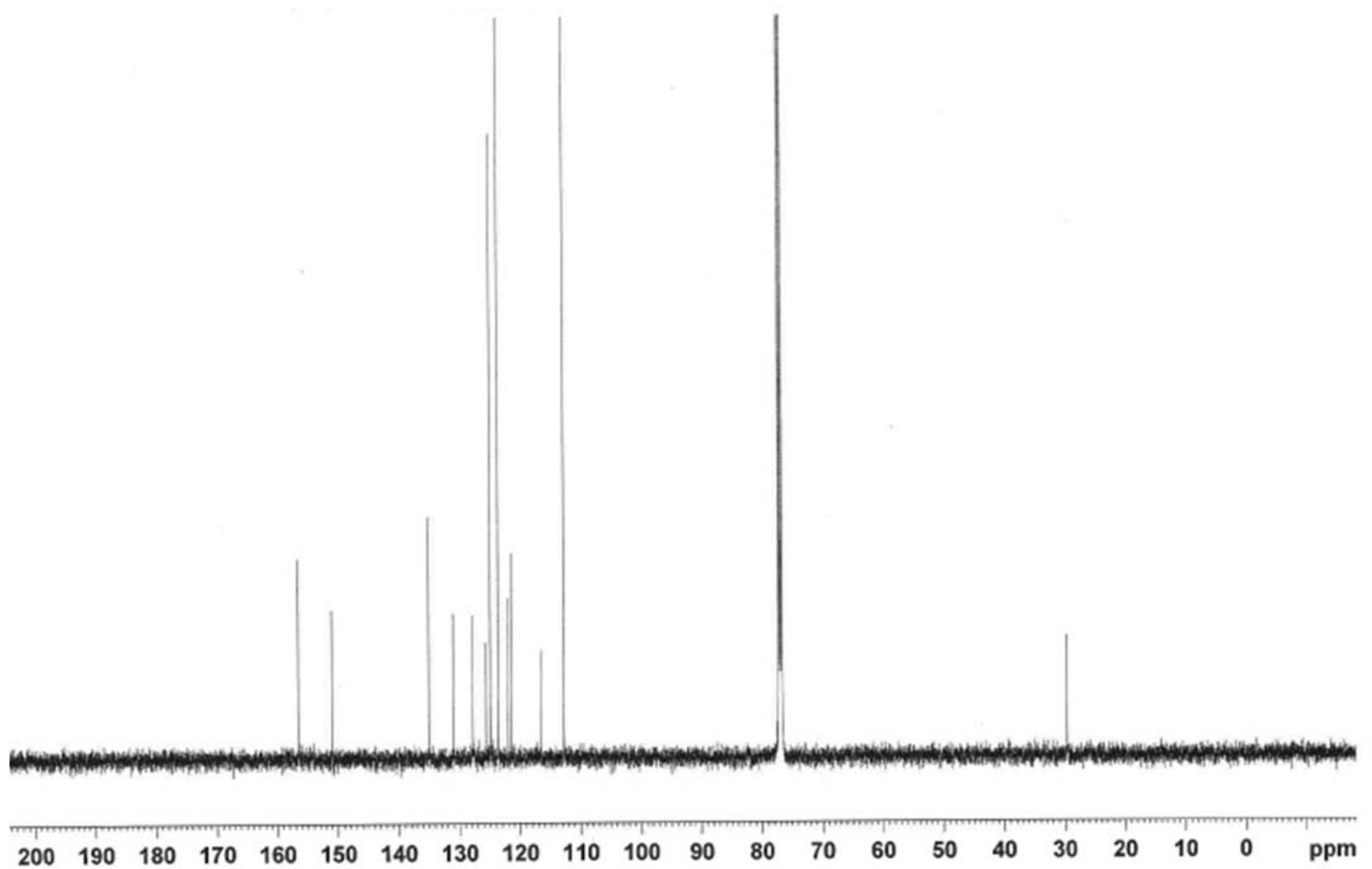




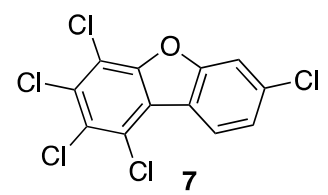

${ }^{1}$ H NMR

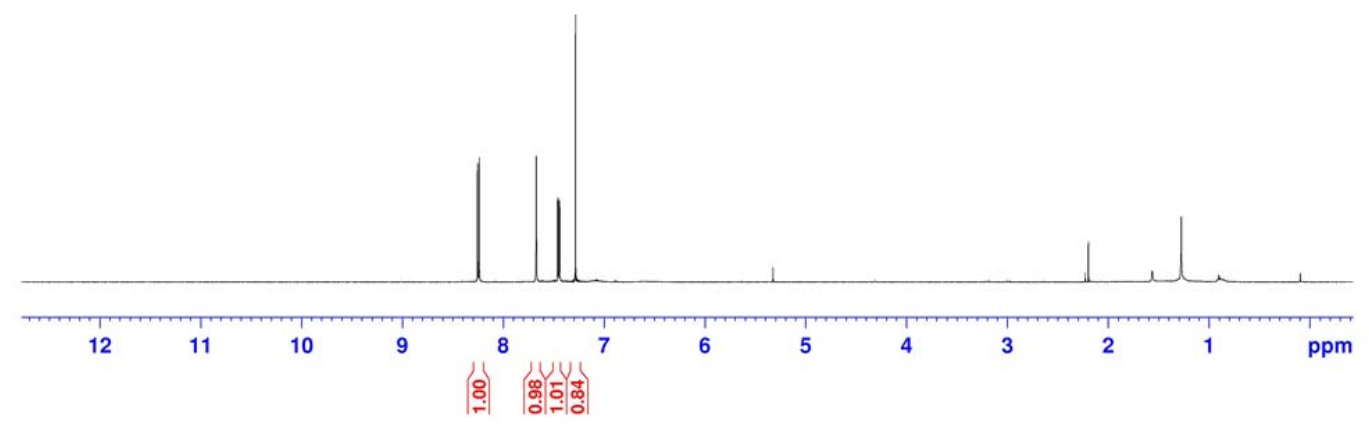




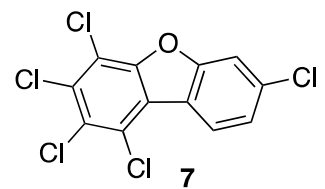

${ }^{13} \mathrm{C} \mathrm{NMR}\left(\mathrm{CDCl}_{3}\right.$ with acetone impurity)

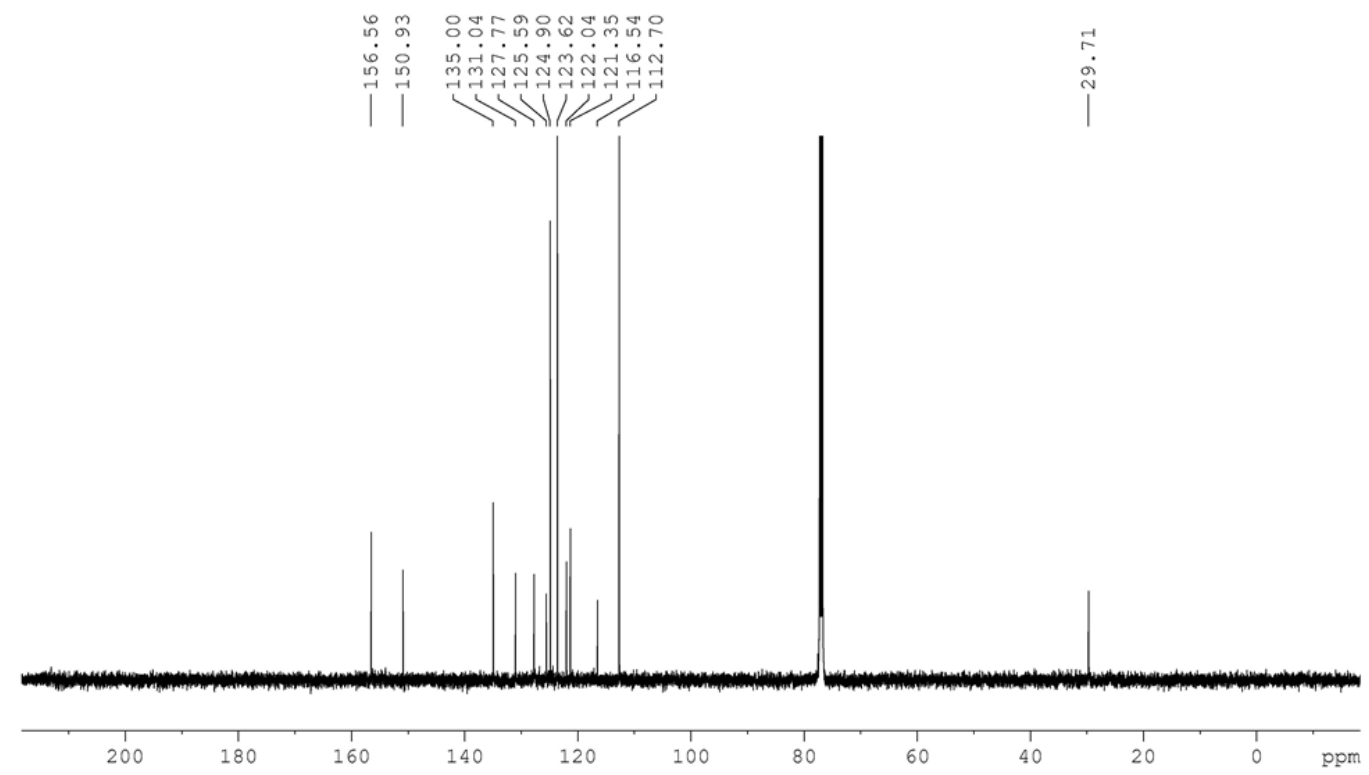




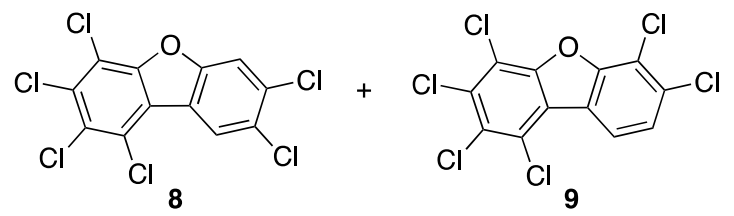

(5:1 ratio)

${ }^{1} \mathrm{H}$ NMR

Proton

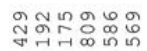

$\infty \infty \infty$

IVIV

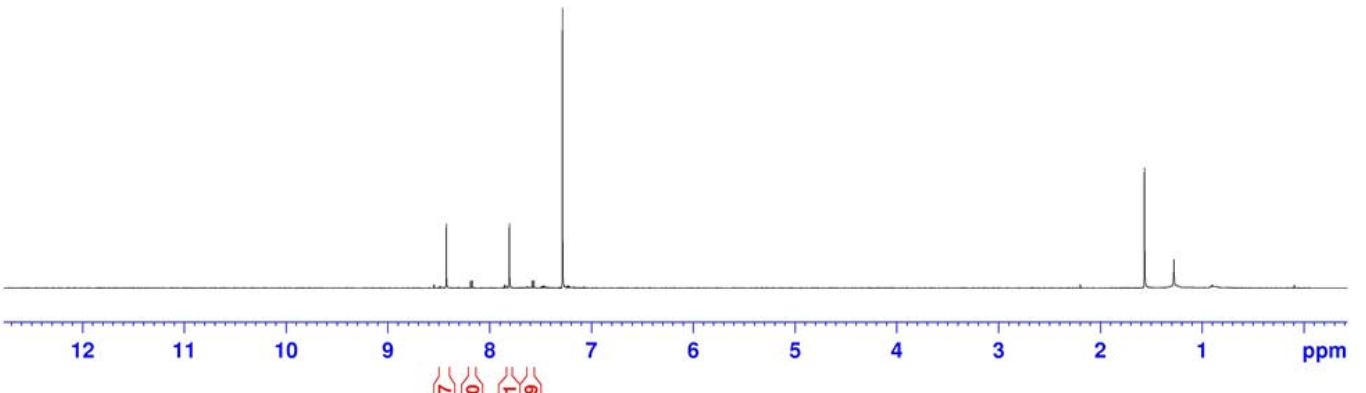

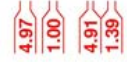


<smiles>Clc1cc2oc3c(Cl)c(Cl)c(Cl)c(Cl)c3c2cc1Cl</smiles>

${ }^{13} \mathrm{C} \mathrm{NMR}\left(\mathrm{CDCl}_{3}\right.$ with acetone impurity)

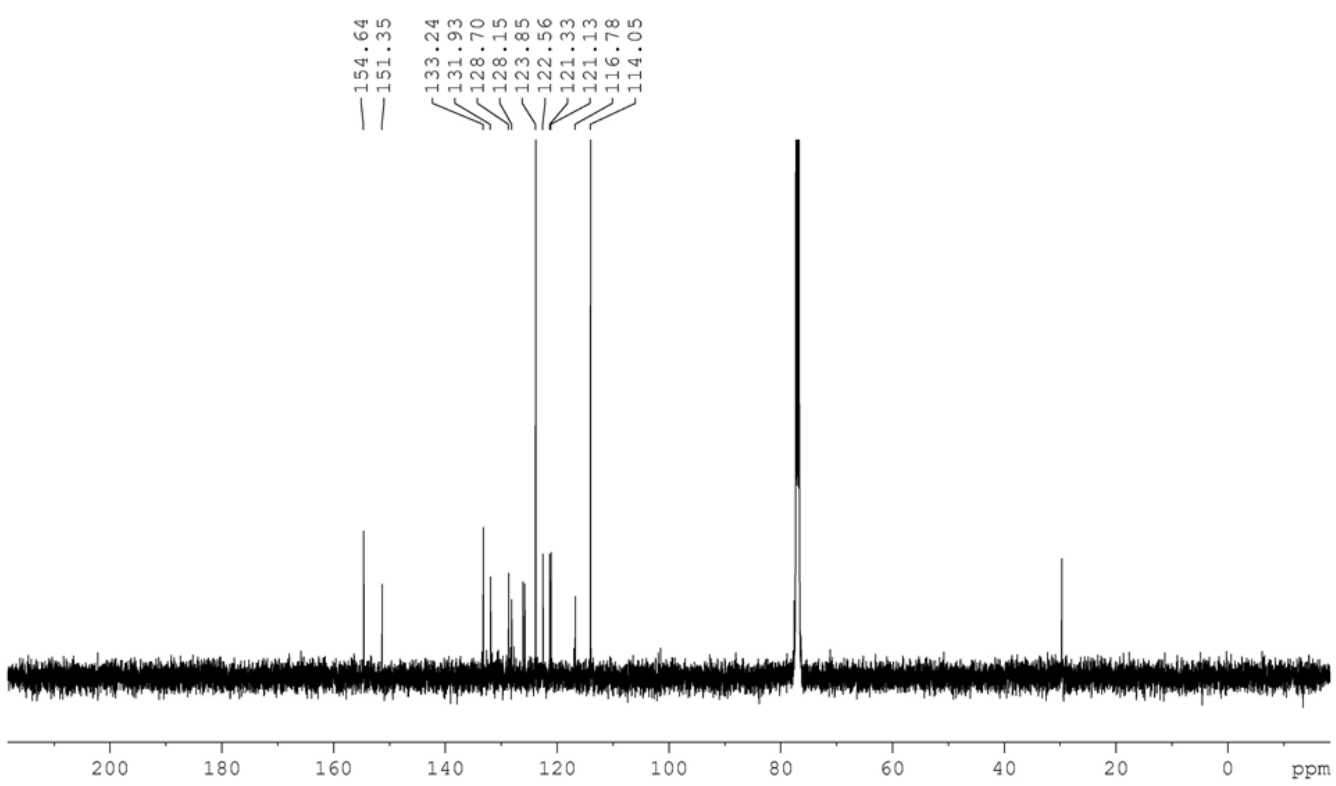




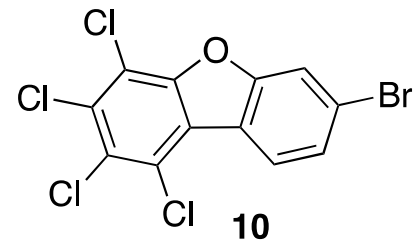

${ }^{1} \mathrm{H}$ NMR

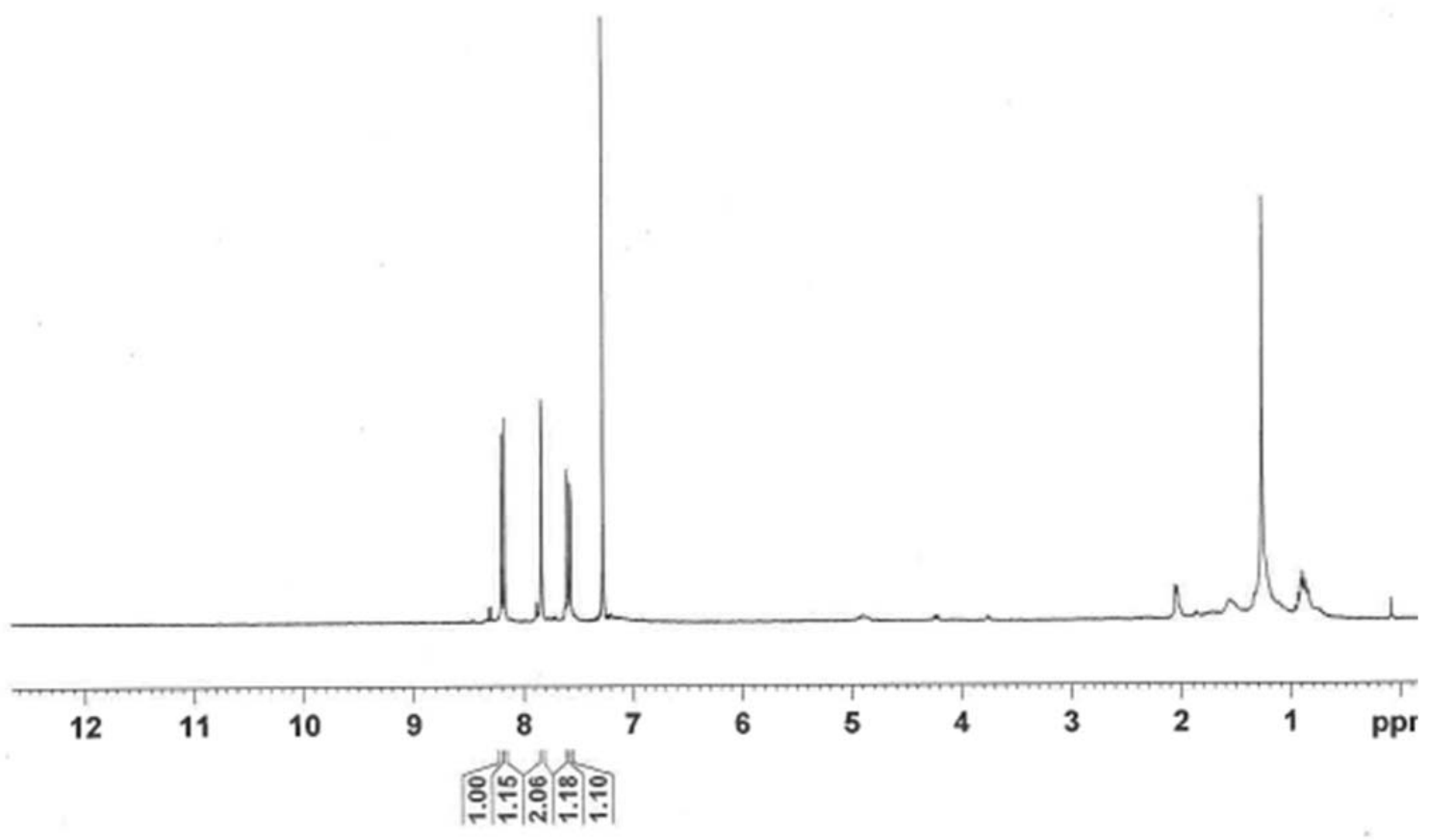


<smiles>Clc1c(Cl)c(Cl)c2c(oc3cc(Br)ccc32)c1Cl</smiles>

${ }^{13} \mathrm{C} \mathrm{NMR}\left(\mathrm{CDCl}_{3}\right.$ with acetone impurity)

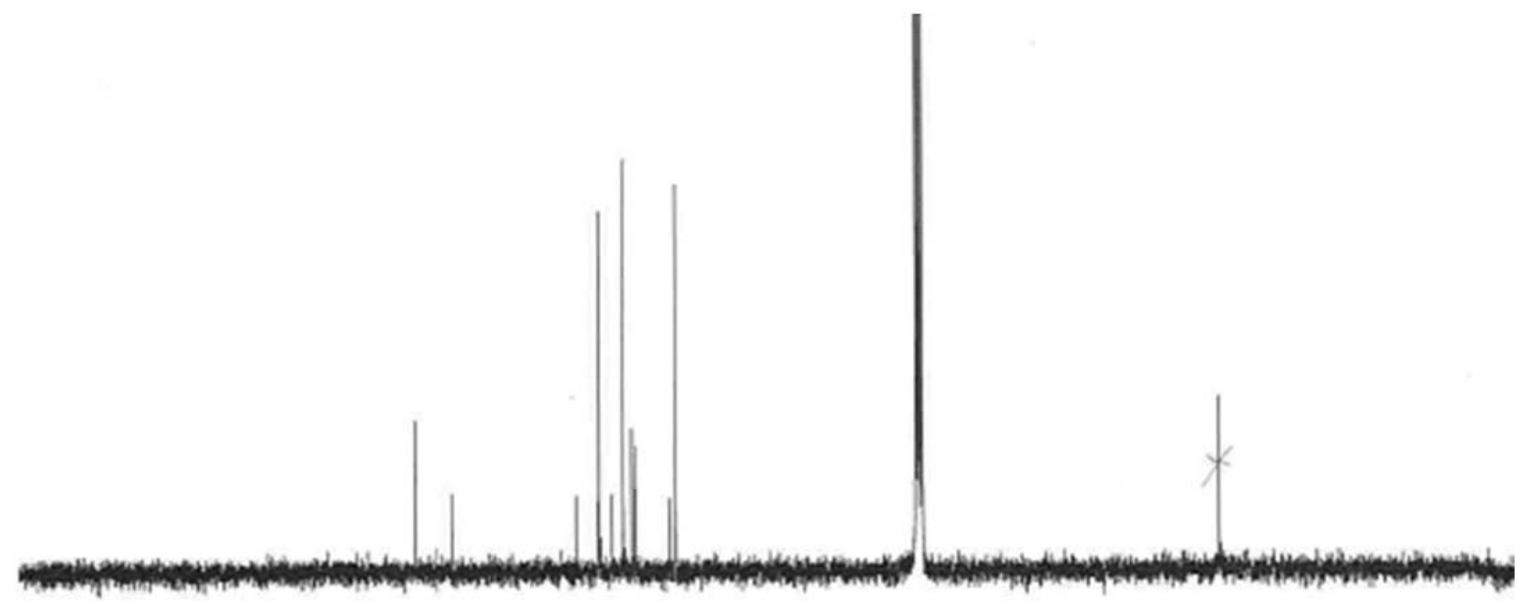

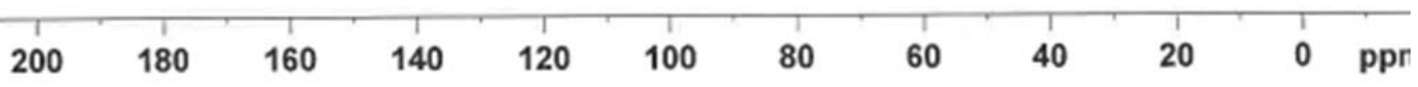




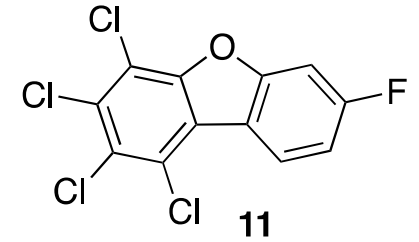

${ }^{1} \mathrm{H}$ NMR

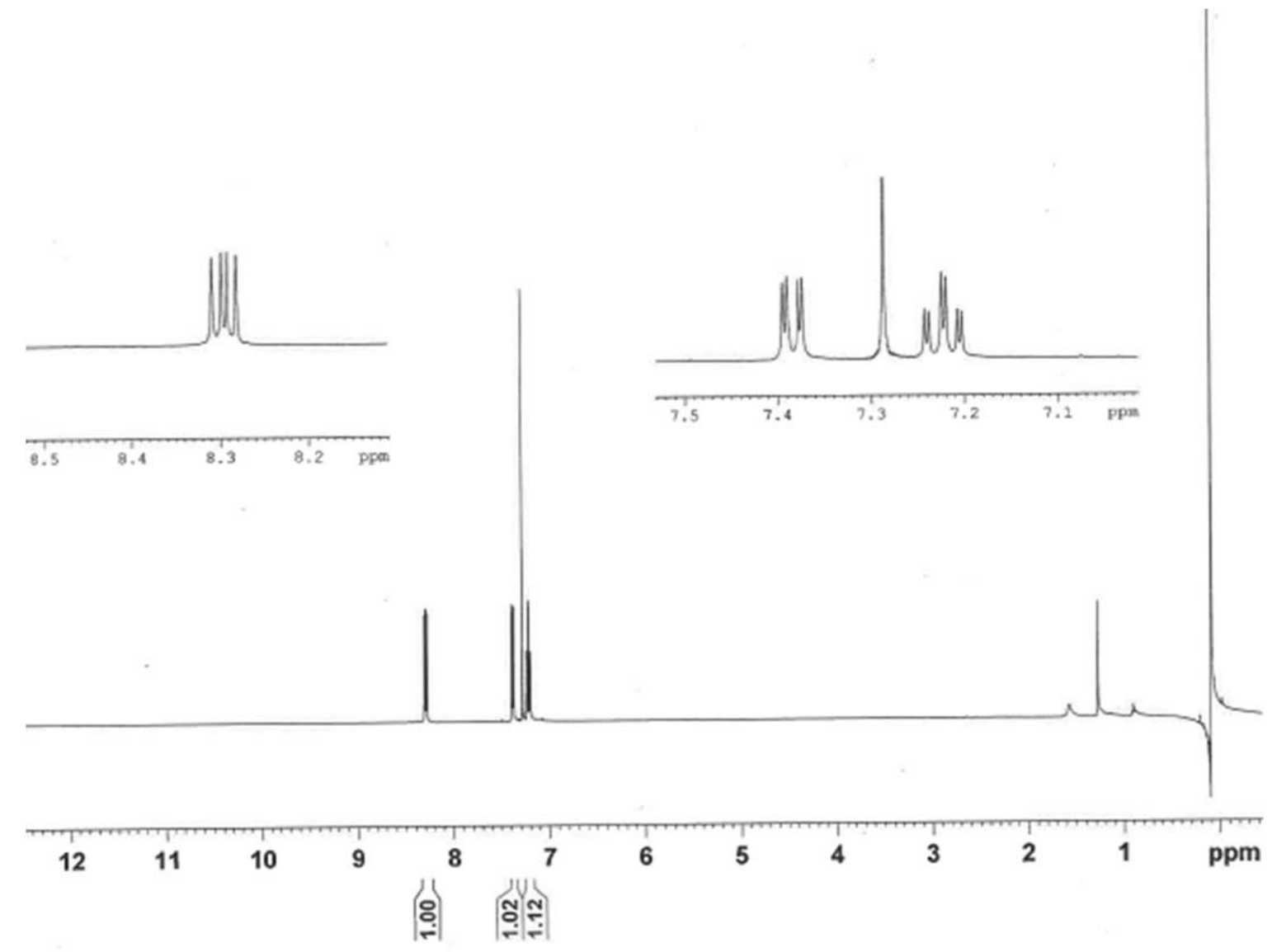




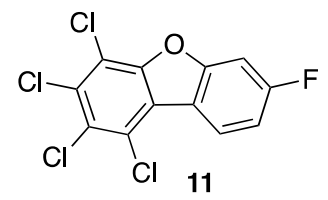

${ }^{13} \mathrm{C} \mathrm{NMR}\left(\mathrm{CDCl}_{3}\right.$ with acetone impurity)

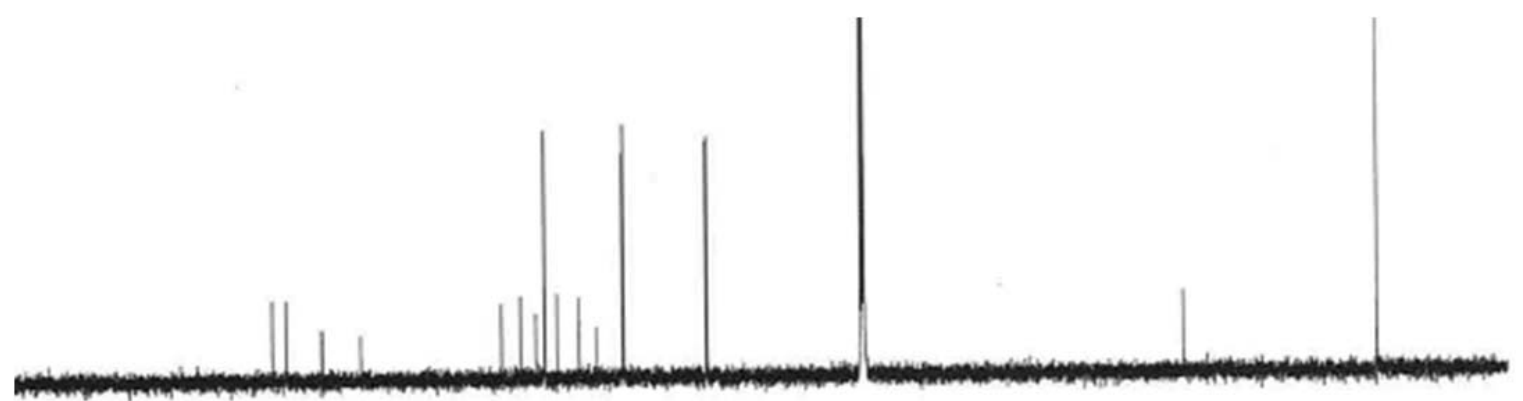

\begin{tabular}{|c|c|c|c|c|c|c|c|c|c|c|}
\hline & 180 & 160 & 140 & 120 & 100 & 80 & 60 & 40 & 20 & ppm \\
\hline
\end{tabular}


Computation methods:

Calculations were done using the Gaussian09W program package. ${ }^{1}$ Geometry optimizations were done using density function theory (DFT) and the hybrid functional B3LYP at the 6-311 $\mathrm{G}^{* *}$ level. ${ }^{2,3}$ Vibrational analyses were performed at the B3LYP/6-311G** level of theory. Zero-point-energy corrections are unscaled.

\section{Literature Citations:}

(1) Gaussian 09, Revision E.01, Frisch, M. J.; Trucks, G. W.; Schlegel, H. B.; Scuseria, G. E.; Robb, M. A.; Cheeseman, J. R.; Scalmani, G.; Barone, V.; Mennucci, B.; Petersson, G. A.; Nakatsuji, H.; Caricato, M.; Li, X.; Hratchian, H. P.; Izmaylov, A. F.; Bloino, J.; Zheng, G.; Sonnenberg, J. L.; Hada, M.; Ehara, M.; Toyota, K.; Fukuda, R.; Hasegawa, J.; Ishida, M.; Nakajima, T.; Honda, Y.; Kitao, O.; Nakai, H.; Vreven, T.; Montgomery, J. A., Jr.; Peralta, J. E.; Ogliaro, F.; Bearpark, M.; Heyd, J. J.; Brothers, E.; Kudin, K. N.; Staroverov, V. N.; Kobayashi, R.; Normand, J.; Raghavachari, K.; Rendell, A.; Burant, J. C.; Iyengar, S. S.; Tomasi, J.; Cossi, M.; Rega, N.; Millam, J. M.; Klene, M.; Knox, J. E.; Cross, J. B.; Bakken, V.; Adamo, C.; Jaramillo, J.; Gomperts, R.; Stratmann, R. E.; Yazyev, O.; Austin, A. J.; Cammi, R.; Pomelli, C.; Ochterski, J. W.; Martin, R. L.; Morokuma, K.; Zakrzewski, V. G.; Voth, G. A.; Salvador, P.; Dannenberg, J. J.; Dapprich, S.; Daniels, A. D.; Farkas, Ö.; Foresman, J. B.; Ortiz, J. V.; Cioslowski, J.; Fox, D. J. Gaussian, Inc., Wallingford CT, 2009.

(2) (a) Becke, A. D. Phys. Rev. A 1988, 38, 3098. (b) Becke, A. D. J. Chem. Phys. 1993, 98, 5648. (c) Lee, C.; Yang, W.; Parr, R. G. Phys. Rev. B 1988, 37, 785

(3) Raghavachari, K.; Binkley, J. S.; Seegar, R.; Pople, J. A. J. Chem. Phys. 1980, 72, 650. 


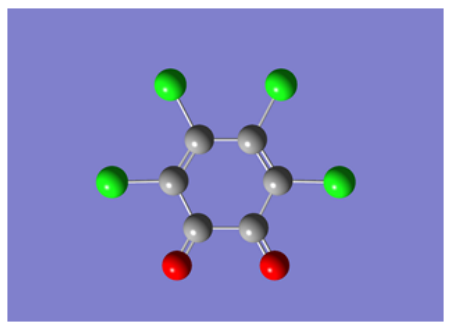

$\begin{array}{lll}\text { Compound } 5 & \text { Imaginary Freq. }=0 \quad \text { Point Group C1 }\end{array}$

B3LYP 6-311G (d,p) optimized structure:

Input orientation:

\begin{tabular}{|c|c|c|c|c|c|}
\hline \multirow{2}{*}{$\begin{array}{l}\text { Center } \\
\text { Number }\end{array}$} & \multirow{2}{*}{$\begin{array}{l}\text { Atomic } \\
\text { Number }\end{array}$} & \multirow{2}{*}{$\begin{array}{l}\text { Atomic } \\
\text { Type }\end{array}$} & \multicolumn{3}{|c|}{ Coordinates (Angstroms) } \\
\hline & & & $x$ & $\mathrm{Y}$ & Z \\
\hline 1 & 6 & 0 & -0.982103 & -0.693880 & -0.056974 \\
\hline 2 & 6 & $\odot$ & $\odot .498923$ & $-\odot .765039$ & -0.056464 \\
\hline 3 & 6 & $\odot$ & 1.277449 & $\odot .583234$ & $-\odot .056922$ \\
\hline 4 & 6 & $\odot$ & $\odot .475439$ & 1.830348 & $-\odot .058090$ \\
\hline 5 & 6 & $\odot$ & -0.879345 & 1.785340 & -0.058548 \\
\hline 6 & 6 & $\odot$ & -1.620391 & $\odot .501970$ & $-\odot .05790 \odot$ \\
\hline 7 & 17 & $\odot$ & 1.371340 & 3.303785 & $-\odot .058828$ \\
\hline 8 & 17 & $\odot$ & -1.798761 & 3.249486 & -0.060003 \\
\hline 9 & 17 & $\odot$ & -3.348080 & $\odot .566321$ & $-\odot .058288$ \\
\hline 10 & 17 & 0 & -1.810348 & -2.206386 & -0.056258 \\
\hline 11 & 8 & $\odot$ & 2.480089 & $\odot .567381$ & $-\odot .056184$ \\
\hline 12 & 8 & 0 & 1.113850 & -1.798675 & -0.055956 \\
\hline
\end{tabular}

Energy + ZPE $=-2219.954618$ Hartree 


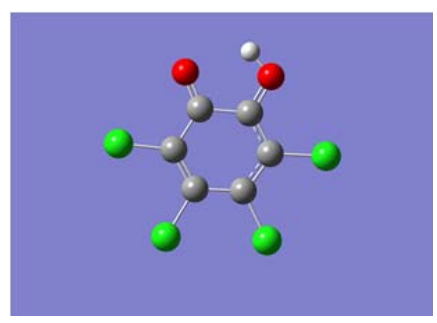

Ion 12a Imaginary Freq. $=0 \quad$ Point Group C1

B3LYP 6-311G (d,p) optimized structure:

Standard orientation:

\begin{tabular}{|c|c|c|c|c|c|}
\hline \multirow{2}{*}{$\begin{array}{l}\text { Center } \\
\text { Number }\end{array}$} & \multirow{2}{*}{$\begin{array}{l}\text { Atomic } \\
\text { Number }\end{array}$} & \multirow{2}{*}{$\begin{array}{l}\text { Atomic } \\
\text { Type }\end{array}$} & \multicolumn{3}{|c|}{ Coordinates (Angstroms) } \\
\hline & & & $\mathrm{X}$ & $\mathrm{Y}$ & Z \\
\hline 1 & 6 & $\odot$ & 1.483032 & $\odot .329105$ & -0.000005 \\
\hline 2 & 6 & 0 & 0.857309 & 1.567319 & -0.000005 \\
\hline 3 & 6 & 0 & -0.664497 & 1.694848 & 0.000023 \\
\hline 4 & 6 & $\odot$ & -1.461917 & $\odot .450730$ & 0.000004 \\
\hline 5 & 6 & 0 & $-\odot .815874$ & -0.750936 & 0.000003 \\
\hline 6 & 6 & $\odot$ & $\odot .658784$ & $-\odot .809819$ & $\odot .0 \odot \odot \odot 18$ \\
\hline 7 & 17 & 0 & -3.148015 & 0.630625 & -0.000009 \\
\hline 8 & 17 & 0 & -1.701104 & -2.215306 & -0.000017 \\
\hline 9 & 17 & $\odot$ & 1.390295 & -2.325180 & $\odot .00 \odot \odot 26$ \\
\hline 10 & 17 & 0 & 3.197808 & 0.248387 & -0.000022 \\
\hline 11 & 8 & $\odot$ & -1.108104 & 2.815370 & 0.000023 \\
\hline 12 & 8 & $\odot$ & 1.510473 & 2.675646 & $-\odot .000 \odot \odot 8$ \\
\hline 13 & 1 & $\odot$ & ๑.877295 & 3.429452 & $\odot .000 \odot 29$ \\
\hline
\end{tabular}

Energy $+\mathrm{ZPE}=-2220.262610$ Hartree 


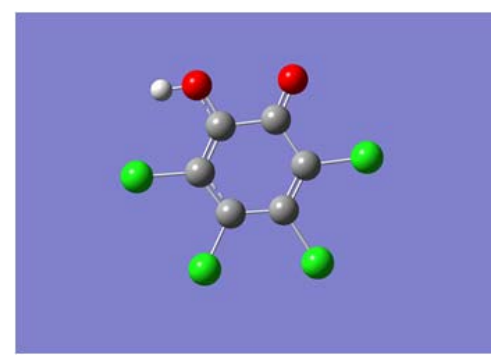

Ion 12b Imaginary Freq. $=0 \quad$ Point Group C1

B3LYP 6-311G (d,p) optimized structure:

Standard orientation:

\begin{tabular}{|c|c|c|c|c|c|}
\hline \multirow{2}{*}{$\begin{array}{l}\text { Center } \\
\text { Number }\end{array}$} & \multirow{2}{*}{$\begin{array}{l}\text { Atomic } \\
\text { Number }\end{array}$} & \multirow{2}{*}{$\begin{array}{c}\text { Atomic } \\
\text { Type }\end{array}$} & \multicolumn{3}{|c|}{ Coordinates (Angstroms) } \\
\hline & & & $x$ & Y & Z \\
\hline 1 & 6 & 0 & -1.458758 & 0.301953 & -0.000004 \\
\hline 2 & 6 & $\odot$ & $-\odot .892437$ & 1.566507 & -0.000030 \\
\hline 3 & 6 & $\odot$ & $\odot .624013$ & 1.742765 & 0.000009 \\
\hline 4 & 6 & $\odot$ & 1.445873 & ๑. 489998 & -0.000036 \\
\hline 5 & 6 & $\odot$ & 0.841052 & -0.734959 & -0.000016 \\
\hline 6 & 6 & $\odot$ & -0.616418 & $-\odot .826534$ & $\odot . \odot \odot \odot \odot \odot ९$ \\
\hline 7 & 17 & 0 & 3.121675 & $\odot .708676$ & ๑. 000003 \\
\hline 8 & 17 & $\odot$ & 1.769162 & -2.177707 & $\odot .0 \odot \odot \odot 27$ \\
\hline 9 & 17 & $\odot$ & -1.324885 & -2.354632 & $-0.000 \odot \odot 7$ \\
\hline 10 & 17 & 0 & -3.185253 & $\odot .176586$ & -0.000025 \\
\hline 11 & 8 & $\odot$ & 1.090294 & 2.845239 & -0.000056 \\
\hline 12 & 8 & $\odot$ & -1.542969 & 2.681801 & $\odot .0 \odot \odot \odot 53$ \\
\hline 13 & 1 & 0 & -2.510411 & 2.545594 & 0.000457 \\
\hline
\end{tabular}

Energy + ZPE $=-2220.256963$ Hartree 


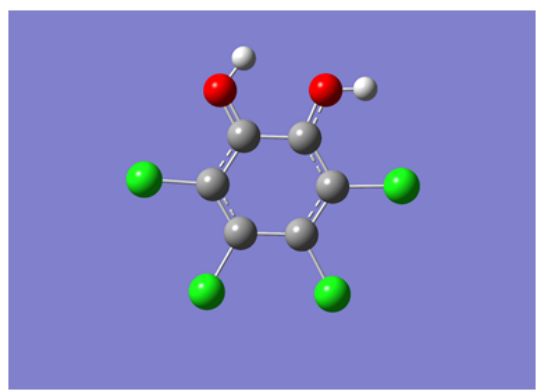

Ion 13a Imaginary Freq. $=0$
Point Group C1

B3LYP 6-311G (d,p) optimized structure:

Standard orientation:

\begin{tabular}{|c|c|c|c|c|c|}
\hline \multirow{2}{*}{$\begin{array}{l}\text { Center } \\
\text { Number }\end{array}$} & \multirow{2}{*}{$\begin{array}{l}\text { Atomic } \\
\text { Number }\end{array}$} & \multirow{2}{*}{$\begin{array}{c}\text { Atomic } \\
\text { Type }\end{array}$} & \multicolumn{3}{|c|}{ Coordinates (Angstroms) } \\
\hline & & & $\mathrm{X}$ & $\mathrm{Y}$ & Z \\
\hline 1 & 6 & $\odot$ & 1.486109 & $\odot .334732$ & $-\odot .000081$ \\
\hline 2 & 6 & $\odot$ & 0.799689 & 1.541655 & 0.000019 \\
\hline 3 & 6 & $\odot$ & -0.710426 & 1.587206 & $\odot .000179$ \\
\hline 4 & 6 & $\odot$ & -1.478579 & $\odot .395052$ & $\odot .000141$ \\
\hline 5 & 6 & 0 & -0.790712 & $-\odot .809979$ & 0.000026 \\
\hline 6 & 6 & 0 & 0.721526 & -0.845836 & -0.000059 \\
\hline 7 & 17 & 0 & -3.172218 & 0.514544 & 0.000254 \\
\hline 8 & 17 & 0 & -1.624443 & -2.258616 & 0.000027 \\
\hline 9 & 17 & $\odot$ & 1.485904 & -2.316708 & -0.000222 \\
\hline 10 & 17 & $\odot$ & 3.198272 & $\odot .354299$ & $-\odot .000227$ \\
\hline 11 & 8 & 0 & -1.296547 & 2.717535 & 0.000063 \\
\hline 12 & 8 & $\Theta$ & 1.316360 & 2.724052 & 0.000121 \\
\hline 13 & 1 & $\Theta$ & -0.710360 & 3.505917 & $-\odot .000038$ \\
\hline 14 & 1 & 0 & 2.298478 & 2.754571 & ๑. $00 \odot \odot 83$ \\
\hline
\end{tabular}

Energy + ZPE $=-2220.397251$ Hartree 


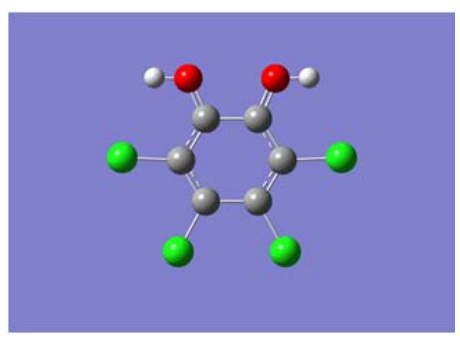

Ion 13b Imaginary Freq. $=0 \quad$ Point Group C1

B3LYP 6-311G (d,p) optimized structure:

Standard orientation:

\begin{tabular}{|c|c|c|c|c|c|}
\hline \multirow{2}{*}{$\begin{array}{l}\text { Center } \\
\text { Number }\end{array}$} & \multirow{2}{*}{$\begin{array}{l}\text { Atomic } \\
\text { Number }\end{array}$} & \multirow{2}{*}{$\begin{array}{c}\text { Atomic } \\
\text { Type }\end{array}$} & \multicolumn{3}{|c|}{ Coordinates (Angstroms) } \\
\hline & & & $\mathrm{X}$ & $\mathrm{Y}$ & Z \\
\hline 1 & 6 & $\odot$ & 1.475612 & $\odot .364629$ & $\odot .000 \odot 12$ \\
\hline 2 & 6 & $\odot$ & 0.757392 & 1.574679 & 0.000028 \\
\hline 3 & 6 & $\odot$ & $-\odot .757394$ & 1.574679 & $-\odot .000274$ \\
\hline 4 & 6 & $\odot$ & -1.475613 & $\odot .364629$ & $-\odot .000130$ \\
\hline 5 & 6 & $\odot$ & $-\odot .758334$ & -0.826436 & 0.000113 \\
\hline 6 & 6 & $\odot$ & 0.758335 & -0.826435 & -0.000087 \\
\hline 7 & 17 & $\Theta$ & -3.185708 & 0.441759 & -0.000203 \\
\hline 8 & 17 & $\odot$ & -1.556255 & -2.288118 & $\odot .000242$ \\
\hline 9 & 17 & $\odot$ & 1.556258 & -2.288118 & -0.000148 \\
\hline 10 & 17 & $\Theta$ & 3.185707 & 0.441763 & $\odot .000069$ \\
\hline 11 & 8 & $\Theta$ & -1.265480 & 2.743687 & 0.000358 \\
\hline 12 & 8 & $\odot$ & 1.265478 & 2.743686 & -0.000091 \\
\hline 13 & 1 & $\Theta$ & 2.249799 & 2.761346 & $-\odot .000082$ \\
\hline 14 & 1 & $\odot$ & -2.249801 & 2.761347 & 0.000637 \\
\hline
\end{tabular}

Energy $+\mathrm{ZPE}=-2220.399788$ Hartree 